\title{
Evaluation of Radio Communication System for sharing location information between traffic objects
}

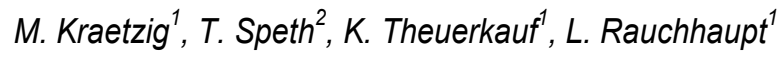 \\ ${ }^{1}$ Institut für Automation und Kommunikation e.V. Magdeburg, Werner-Heisenberg-Str. 1, 39106 Magdeburg, \\ Germany, marko.kraetzig@ifak.eu \\ ${ }^{2}$ Technische Hochschule Ingolstadt, CARISSMA, Paradeplatz 13, 85049 Ingolstadt, Germany, \\ Thomas.Speth@thi.de
}

\begin{abstract}
:
Dedicated short range communication (DSRC) or radio systems for intelligent transport systems (ITS) should be used for transmission of position information between dynamic traffic objects. The position information shall be used to improve the accuracy of localisation of traffic objects. Therefore the safety in road traffic is increased by avoiding dangerous situations and accidents. Due to relative movement of traffic objects the position data becomes outdated, the longer it takes to transmit the information via ITS radio system. For that reason, the transmission time of ITS radio system shall provide an input to correct the transmitted position data at the receiver.
\end{abstract}

Keywords: Intelligent Transport Systems, System analysis, Transmission characteristics, Timing analysis, Performance analysis, Performance characteristics, Reliability analysis, Reliability evaluation

\section{INTRODUCTION}

This paper starts with a description of the application scenario of time critical communication between intelligent transport systems (ITS). Thereafter a model is introduced from which relevant characteristic parameters are derived which are used to assess reliability. The test method to evaluate the performance of ITS radio system is also part of the report. The main part of the paper is the performance assessment of radio system for intelligent transport systems (ITS). The focus is the assessment of time and error behaviour. Furthermore, the effects of influencing parameters will be shown by measurements results. At the end of the paper the results of evaluation will be discussed.

\section{APPLICATION SCENARIO}

Applications for integral vehicle safety as well as some advanced driver assistance systems (ADASs) benefit from precisely determination of relative positions between dynamic traffic objects. Especially the intervehicular exchange of position and dynamic data offers a new potential for these systems, e.g. vehicleinteractive applications [1]. Compared to conventional environmental sensors like Radar or Lidar, there are two major benefits: there is no limitation of angle of beam spread, i.e. vehicle-to-vehicle (V2V) communication acts like a $360^{\circ}$ field of view sensor. Second, it works in non-line-of-sight conditions and thus an autonomous safety system can react earlier. For this purpose, a vehicle's self-positioning has to better than lane-level accuracy [2].

However, with conventional global navigations satellite system (GNSS) measurements, e.g. using Global Positioning System (GPS), the required accuracy cannot be achieved [3]. Known methods for improved positioning are Differential GNSS (DGNSS) or RealTime Kinematic (RTK). Both techniques lean on GNSS observation data of a nearby reference station.

Focusing on RTK, the typical accuracy is below $0.1 \mathrm{~m}$ RMS. To achieve this accuracy, a moving GNSS receiver, called rover, calculates double differences out of its own carrier phase measurements and the received one from a reference station. The result of this calculation is a base line vector between both receivers. With the well-known position of the reference station, it is trivial to get a high accurate position of the rover in global reference frame. [4]

However, the reference station operator often charges for these observation data and also the data link, often established by cellular mobile communication, causes 
costs. For this reason, we propose, instead of a stationary reference station, a moving base, which can be any other vehicle in the vicinity of the ego-vehicle. By this reduced RTK method, no absolute positioning is possible anymore, thus it is not necessary for the moving base sending their position information. Sending the GNSS observation suffices to calculate a base line vector. Future vehicle safety system might benefit from this cooperative relative positioning method. Similar approaches can be found in [5], [6], [7].

With growing transmission time, however, the accuracy is decreasing, because the base line vector is calculated between the two points of moving base and rover at that the observation were taken. Thus, with larger differential age between both observation data, the calculated relative position becomes obsolete and inaccurate. In this paper we analyse this error source so it can be considered and corrected in further works.

\section{MODEL}

\subsection{Requirements}

First we would like to clarify what reliability means in context of wireless communication for intelligent transport systems. A user of a wireless communication system expects a certain value, e.g. position or velocity, at a certain interface within a defined time frame without any errors under defined conditions. This is an informal definition. In order to be able to assess the degree of fulfilment of this requirement by means of simulation or measurement, a formal model is required.

First of all this model has to take into account the application field - the wireless communication for ITSs. The parameters to be investigated have to be in line with the design criteria of wireless communication systems. Parameters such as Data Throughput or Bit Error Rate are normally not useful to design a particular wireless application which e.g. shall transmit time, critical localisation and motion data information.

Furthermore, the model has to consider that there is no standard interface between communication and application available. Last but not least the model should represent the conditions of reality as accurate and complete as possible and necessary. The following section introduces an approach which fulfils the mentioned requirements.

\subsection{Approach}

The abstraction of a localisation application using wireless communication for ITSs is shown in Fig. 1. The wireless communication modules are seen as an internal or external part of localisation devices. The localisation devices have to fulfil certain functions in distributed moving traffic objects and therefore they have to communicate via wireless communication media. From the point of view of the ITSs, the communication characteristics at the interface provided by the wireless solution are important. These communication characteristics have to fit to the time and error categories.

The communication interface must be clearly defined, upon which the characteristics are related to. This interface consists of a hardware part such as Ethernet and a software part such as a communication protocol or an application interface. Besides a clear statement concerning the communication interface and the communication characteristics, the conditions have to be described under which the characteristic values are valid. The conditions can be described by a number of influencing values which have different origins. It is obvious that the communication system itself affects the characteristics concerning e.g. data rate. It is also evident that the communication media has influence because of other users of the spectrum or because of the effects of fading effects. Furthermore, the characteristics depend on the options chosen in the devices, which means on its configuration. It is sometimes forgotten that also the application affects the characteristic values in the sense of the size of a packet or the cycle of requests on the communication system.

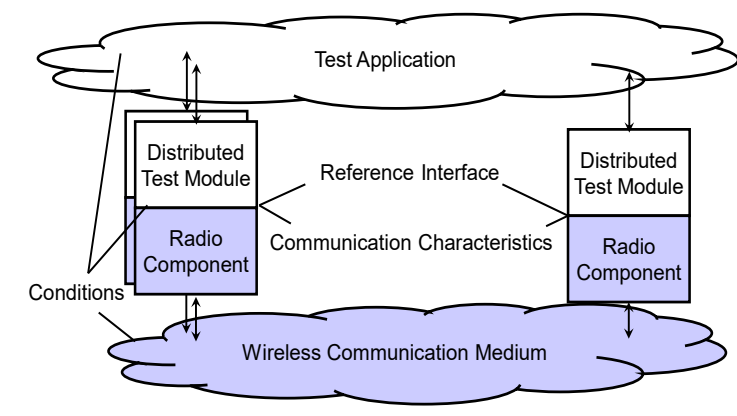

Fig. 1: Model approach for the assessment of wireless communication systems.

\section{CHARACTERISTIC PARAMETERS}

\subsection{Definitions}

\section{Reliability}

Now we can define the term reliability more specific and we can describe how to assess reliability. In line with the definition of chapter 2.1, reliability can be seen as the degree in which you can expect that a wireless communication solution meets the limits of relevant characteristic parameters. With this definition it is obvious that the assessment of reliability requires stochastic methods. The characteristic parameters are random variables. Their behaviour follows probability density functions. The reliability is the probability that a value of a characteristic parameter is less or equal to the limit defined by the application.

The reliability according VDI 4001 [8] is a comprehensive term to describe the availability with corresponding influencing parameters functionality, maintainability and supportability capabilities. 
Therefore, the reliability is no measurable characteristic parameter.

\section{Availability}

The availability according VDI 4001 [8] is the ability of a unit to perform a required function at a given time or during a given time interval. The meaning in the context of wireless communication for intelligent transport systems is that the wireless device transfer application relevant user data from the producer to the consumer and the opposite direction. The required functions are an unchanged content of user data and a user data transfer within a prescribed period of time or at a required point of time.

\section{Maintainability}

The maintainability according VDI 4001 [8] is the ability of a unit under the given conditions may be left in a state or restore to the state in which it can perform the required functions. The meaning in the context of wireless communication for intelligent transport systems is that the wireless device provides procedures to observe system condition and to detect incipient deviations from that condition. Furthermore the wireless devices provide mechanisms to restore the previous status automatically. Wireless solutions use mechanisms like forward error correction, cyclic redundancy check, transmission repetition or multiple transmissions.

\subsection{Characteristic parameters to assess the time behaviour}

The characteristic parameters to assess the time behaviour of wireless systems are set out in this subsection. VDI 2185 [9] define characteristic parameters for the assessment of radio based communication in industrial automation. The characteristic parameters "Transmission Time" and "Packet Lost Rate" according to [9] are adequate for the assessment of time and error behaviour of wireless devices for intelligent transport systems.

\section{Transmission Time}

The transmission time according to [9] is a very important characteristic parameter, because:

- Real-time location applications are difficult or impossible with unforeseeable deviations of transmission time

- High values of transmission time are a problem for high-precision localisation algorithms

The definition of the transmission time according [9] is based on a producer-consumer-model (see Fig. 2). It is the time duration from the handing over of the first user data byte of a packet at the reference interface in the test producer, up to the handing over of the last user data byte of the same packet at the reference interface at the test consumer.

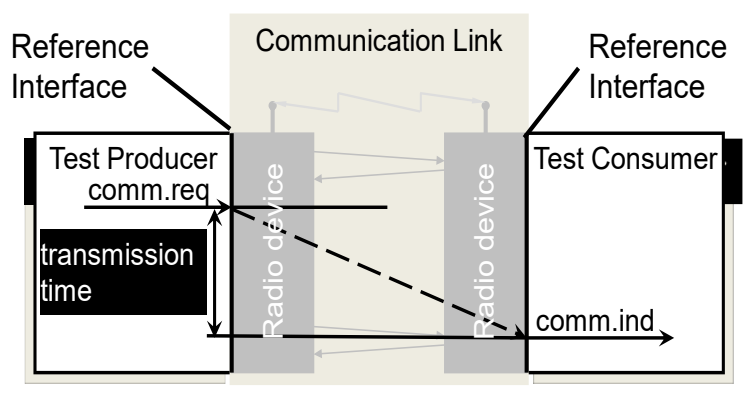

Fig. 2: Definition of the transmission time. [9]

\section{Packet Loss Rate}

The packet loss rate (PLR) is ascertained according to the producer-consumer-model. It reveals, how many of the packets, transferred from the application to the communication interface within the producer, are transmitted from the communication interface to the application within the consumer. The packet loss rate is determined as follows in equation (1):

$$
\text { PLR }=\frac{N_{T x}-N_{R x}}{N_{T x}}=\frac{N_{P L}}{N_{T x}}=1-A
$$

Whereby:

$$
\begin{aligned}
& -N_{T x} \text { means transmitted packets } \\
& -N_{R x} \text { means received packets } \\
& -N_{P L} \text { means number of lost packets }
\end{aligned}
$$

The number of lost packets (NPL) is determined by three cases. The first case of packet loss is that a transmitted packet was not received. The second case of packet loss is that a packet was received after an application related maximum of transmission time $\left(t_{T T \max }\right)$. Moreover a packet received in an incorrect sequence is classified as packet loss. The three cases of lost packets are summarized in equation (2).

$$
N_{P L}=\sum_{i=1}^{N_{R K}} l\left(p_{R x i}\right) \text { with }\left\{\begin{array}{l}
l\left(p_{R x i}\right)=0, \text { other } \\
l\left(p_{R x i}\right)=1, t_{T T}\left(p_{R x i}\right)>t_{T T \max }^{i} \\
l\left(p_{R x i}\right)=1, \sum_{j=1}^{i} t_{T I}\left(p_{R x i j}\right)<\sum_{k=1}^{i-1} t_{T I}\left(p_{R x k}\right)
\end{array}\right.
$$

\section{Availability}

The availability (A) is the ability of a unit to perform a required function at a given time or during a given time interval. The availability is the ratio of correct data transmission time (uptime: $\mathrm{t}_{\mathrm{t}}$ ) to observation time $\left(\mathrm{t}_{\mathrm{o}}\right)$.

$$
A=\frac{t_{U}}{t_{O}}
$$

Under the assumption that the producer or client transmits cyclic user date frames with a fixed transmission interval $\left(t_{T_{1}}\right)$ during the observation period $\left(t_{0}\right)$ and each received frame consider as correct for the 
transmission interval $\left(t_{T_{I}}\right)$. The availability can be calculated as follows in equation (4):

$$
A=\frac{N_{R x} t_{T I}}{N_{T X} t_{T I}}=\frac{N_{R x}}{N_{T X}}
$$

The availability $\left(\mathrm{a}_{\mathrm{i}}\right)$ depends on the number of lost frames for any observation period $\left(\mathrm{t}_{\mathrm{O} i}\right)$ and can be calculated as in the following equation (5):

$$
\mathrm{a}_{\mathrm{i}}\left(\mathrm{t}_{\mathrm{O}}\right)=\frac{\Delta \mathrm{N}_{\mathrm{Rxi}}}{\Delta \mathrm{N}_{\mathrm{TXi}}}=1-\frac{\Delta \mathrm{N}_{\mathrm{PLi}} \mathrm{t}_{\mathrm{TI}}}{\Delta \mathrm{N}_{\mathrm{TXi}} \mathrm{t}_{\mathrm{TI}}}=1-\Delta \mathrm{N}_{\mathrm{PLi}} \frac{\mathrm{t}_{\mathrm{TI}}}{\Delta \mathrm{t}_{\mathrm{oi}}}
$$

The ration between transmission interval $\left(t_{T I}\right)$ and observation period ( $t_{0}$ ) according to equation (5) is particularly important. If the observation period approaches the transmission interval, the number of single lost packets has a much greater effect on availability. But in the opposite direction the influence of lost packets at availability can't recognise with longer observation periods.

\section{TEST ARCHITECTURE}

\subsection{System under test}

The system under test (SUT) consists of two devices under test. The devices under test (DUT) are on-board units (OBU) MK5 from Cohda Wireless (see Fig. 3).

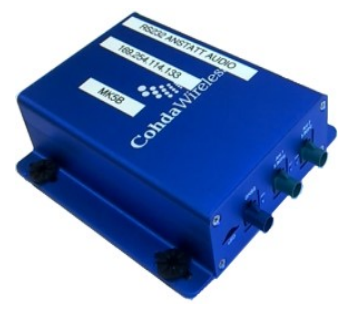

Fig. 3: DUT: On-board Unit MK5

The frequency band for intelligent transport systems (ITS) range from $5.875 \mathrm{GHz}$ to $5.905 \mathrm{GHz}$ (ITS-G5). The ITS-G5B frequency band is specified for ITS nonsafety road traffic applications and the ITS-G5A frequency band is specified for ITS road traffic safety applications and it is only allowed to be used by ITS-G5 compliant stations [10], [11].

Tab. 1: ITS channel allocation

\begin{tabular}{|c|c|c|c|c|}
\hline $\begin{array}{c}\text { ITS } \\
\text { Frequency } \\
\text { Band }\end{array}$ & $\begin{array}{c}\text { Channel } \\
\text { type }\end{array}$ & $\begin{array}{c}\text { Centre } \\
\text { frequency } \\
\text { [MHz] }\end{array}$ & $\begin{array}{c}\text { IEEE } \\
\text { channel } \\
\text { number }\end{array}$ & $\begin{array}{c}\text { E.I.R.P. } \\
\text { limit } \\
\text { [dBm] }\end{array}$ \\
\hline ITS-G5B & G5-SCH4 & 5860 & 172 & 0 \\
\cline { 2 - 5 } & G5-SCH3 & 5870 & 174 & 23 \\
\hline ITS-G5A & G5-SCH1 & 5880 & 176 & 33 \\
\cline { 2 - 5 } & G5-SCH2 & 5890 & 178 & 23 \\
\cline { 2 - 5 } & G5-CCH & 5900 & 180 & 33 \\
\hline
\end{tabular}

The SUT use the service channels G5SC1, which is dedicated for ITS road traffic safety applications and allow an equivalent isotropically radiated power (E.I.R.P.) of $33 \mathrm{dBm}$. Detail of ITS channel allocation can be found Tab. 1. The basic topology of SUT is a point to point topology with two end points. Two physical links are established between the end points, because the DUT use a $2 \times 2$ MIMO. The DUT hardware interfaces, RS232 and Ethernet, are considered as reference interface and all determined characteristic parameters a valid according to the used reference interfaces.

\subsection{Measurement Setup}

The test architecture and measurement setup is depicted in Fig. 4. All testing shall be conducted in a reproducible, shielded area. Therefore the DUT's are placed in a radio frequency (RF) shielded box and the $\mathrm{RF}$ signal is connected via coaxial cable. The radio transmission channel is emulated by a channel emulator and the physical endpoints (PEP_C, PEP_P) of DUT's are connected with this channel emulator. The type of test application depends on the reference interface. An Ethernet quality analyser is used for the measurement of time and error characteristics at the reference interface Ethernet. Therefore the Ethernet quality analyser analyses the Ethernet traffic simultaneously at the reference interface at producer and consumer site. The test data traffic is generated by a PC. A different test application is used for the reference interface RS232. The test data traffic is generated by an embedded device with RS232 interface. The time and error characteristics are measured via a software application within the DUT's. For this purpose the GPS signal is used for time synchronisation between DUT's. But for measurement on the reference interface RS232 only the wireless communication is considered. The serial communication from the embedded device to the DUT is neglected in assessing the SUT. The measured values of characteristic parameters are transferred via Ethernet to a PC and saved in a result file.

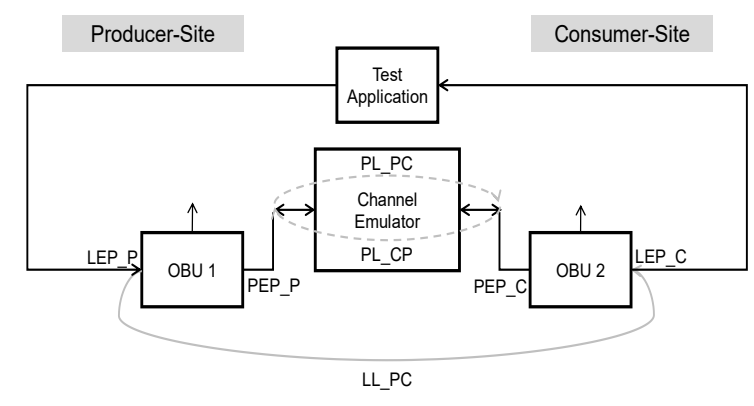

Fig. 4: Measurement setup

The channel emulator emulates the radio transmission channel for a static and an application-related scenario. The static scenario considers a fixed attenuation without moving effects between the DUT's. The application-related scenario considers two cars, each with an OBU. The cars go straight on a street until a 
crossing with traffic light. The cars must reduce speed and stop in front of a red traffic light for a period of $10 \mathrm{~s}$. Thereafter both cars accelerate and the same procedure takes place in reverse order. The applicationrelated scenario is designated as movement scenario below.

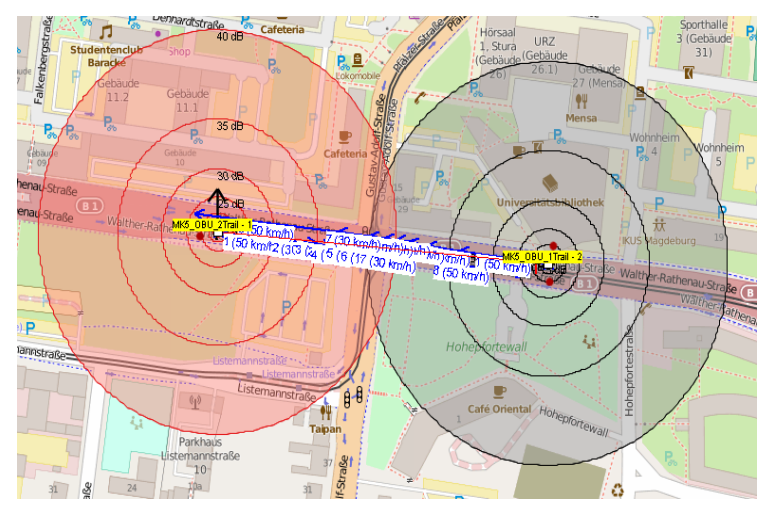

Fig. 5: Measurement setup 2

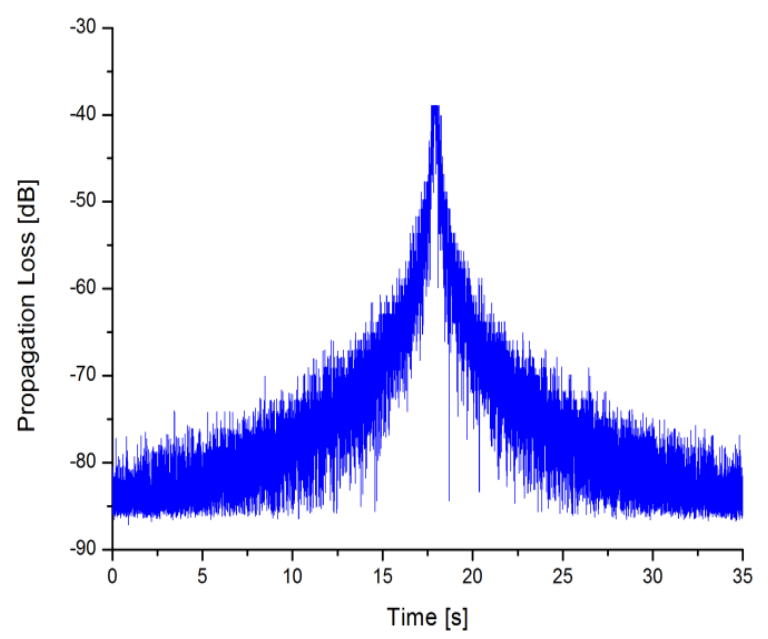

Fig. 6: Propagation loss

The propagation loss between the DUT's (OBU's) is depicted in Fig. 6. The statistical model for the effects of real propagation environment is emulated with a Rician distribution, as we are assuming that the dominant path is available between the two DUT's.

\section{MEASURMENT RESULTS}

To assess the time behaviour of a wireless systems for ITS, the well-known statistical parameters for extreme values (e.g. minimum and maximum value), the most commonly occurring value (e.g. Mode) and other values for the measures of location (e.g. Percentile $95 \%$ or $99 \%$ ) can be used. The maximum value is not qualified for assessment, since it is a single value of a series of measurements and it is not sure that the real maximum value is captured. An infinite measurement of the transmission time would be necessary or an inference to a larger population using methods of interferential statistics. However, the maximum value is considered so far as it influences the percentile value.
The results of transmission time measurements for reference interface Ethernet are depicted in Fig. 7 and Fig. 8.

Tab. 2: Statistical parameters for measurement results with reference interface Ethernet

\begin{tabular}{|c|c|c|c|c|c|}
\hline \multirow{2}{*}{ Scenario } & \multicolumn{4}{|c|}{ Transmission Time [ms] } & \multirow{2}{*}{ PLR } \\
\cline { 2 - 5 } & Min & P95 & P99 & Max & {$[\%]$} \\
\hline Static & 2,2 & 4,4 & 6,3 & 25,5 & 0,0 \\
\hline Movement & 2,2 & 4,1 & 6,0 & 60,8 & 4,4 \\
\hline
\end{tabular}

Tab. 2 lists the statistical parameters for the measurement results with the reference interface Ethernet for the scenario static and movement. There is a minor difference between the measurement results of transmission time in scenario static and movement. But, the number of lost packets is growing for scenario movement. The reason for the lost packets is the random signal attenuation at the maximum distance between the SUT transceiver and receiver. If the constellation is particularly disadvantageous, the link budget (signal level above receiver sensitivity) is too small and packets can't be received.

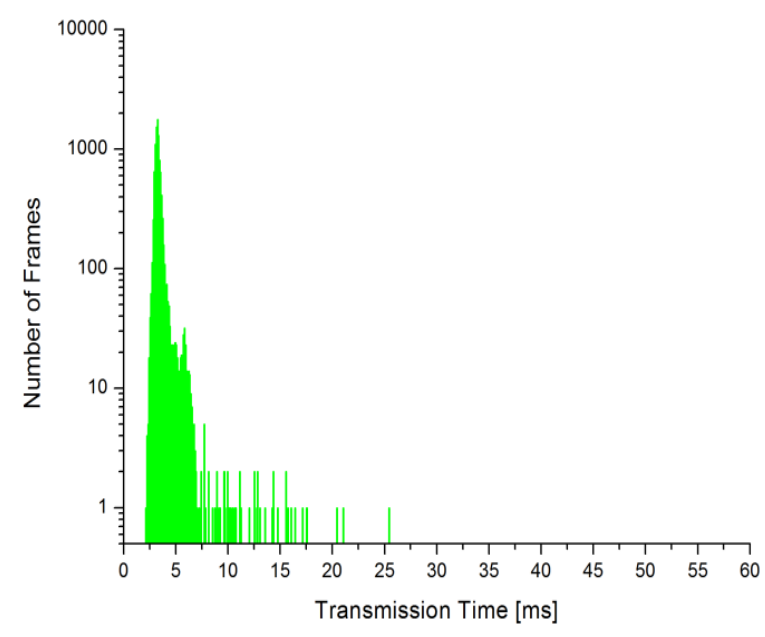

Fig. 7: Static scenario, Reference Interface: Ethernet

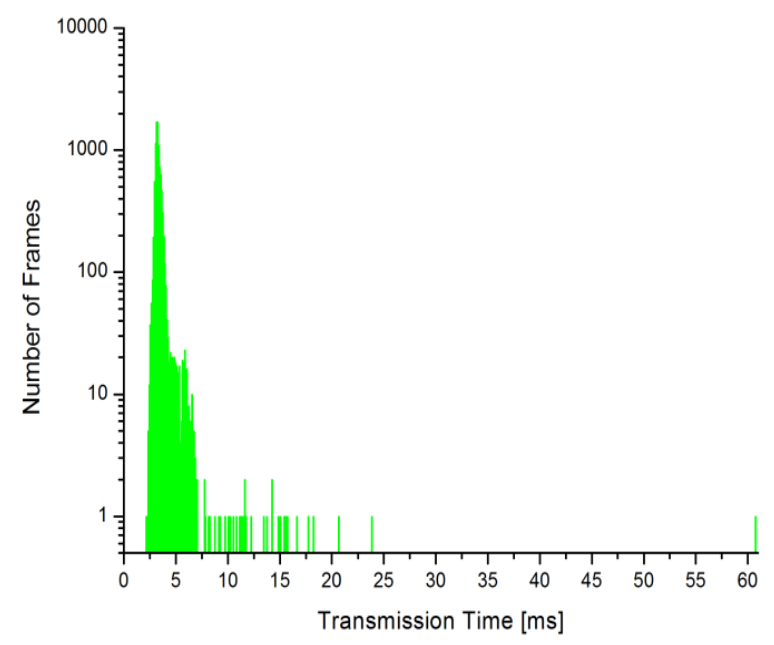

Fig. 8: Moving scenario, Reference Interface: Ethernet 
Tab. 3 lists the statistical parameters for the measurement results with the reference interface RS232. There is also a minor difference between the test results of transmission time in scenario static and movement. The number of lost packets of scenario static with reference interface RS232 is with $5.5 \%$ lost packets relative high in contrast to the measurement results with reference interface Ethernet. The number of lost packets is doubling for scenario movement. The reason for the high number of lost packets of scenario movement is once again the random signal attenuation at a maximum distance between DUT transceiver and receiver. The reason for the lost packets of test case static was not clarified.

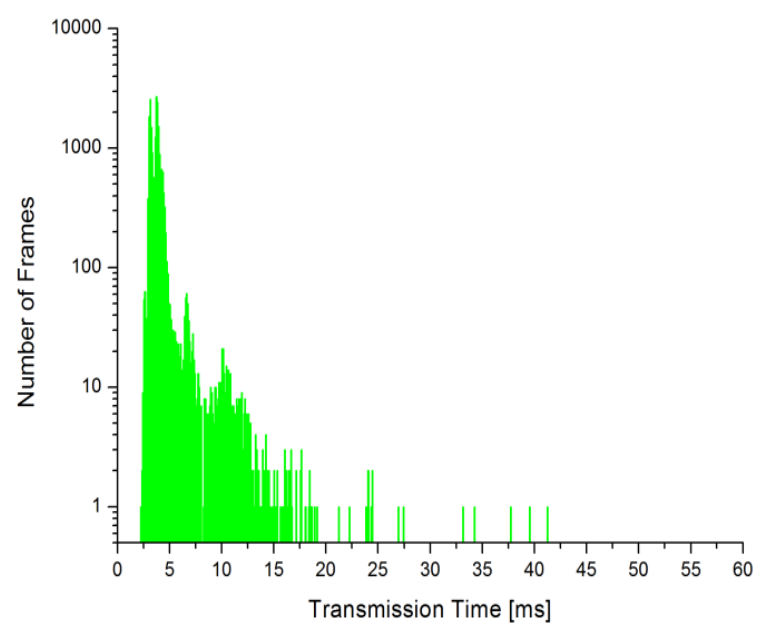

Figure 9: Static scenario, Reference Interface: Ethernet

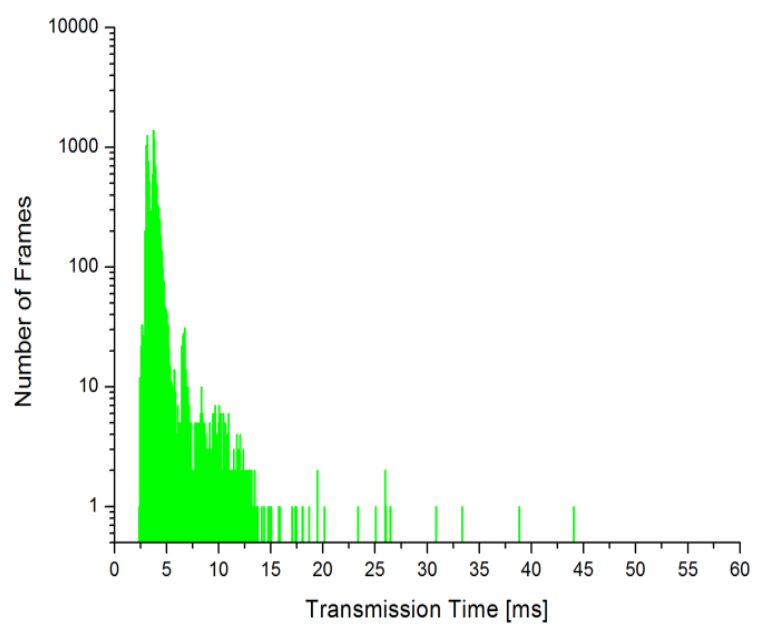

Fig. 10: Static scenario, Reference Interface: Ethernet

Tab. 3: Statistical parameters for measurement results with reference interface $R S 232$

\begin{tabular}{|c|c|c|c|c|c|}
\hline \multirow{2}{*}{ Scenario } & \multicolumn{4}{|c|}{ Transmission Time [ms] } & \multirow{2}{*}{ PLR } \\
\cline { 2 - 5 } & Min & P95 & P99 & Max & {$[\%]$} \\
\hline Static & 2,3 & 5,4 & 10,8 & 41,3 & 5,5 \\
\hline Movement & 2,4 & 5,1 & 10,2 & 44,0 & 10,9 \\
\hline
\end{tabular}

The results of transmission time measurements for reference interface RS232 are depicted in Figure 9 and Fig. 10.

\section{CONCLUSIONS}

In the paper we presented a proposal on how to assess the time and error behaviour of wireless solutions for intelligent transport systems. A fundamental requirement for such a method is the focus on the application. That is why characteristic values such as transmission time and packet loss are used in the way defined in this paper. It was pointed out that these parameters are random variables, which means that the statistical parameters have to be considered. The approach can be used for analytical studies, simulations and tests.

Furthermore the results can be used for the assessment of wireless systems from the point of view of vehicle to vehicle communication for time critical localisation applications. The measurement results can help to estimate the usage of the considered wireless communication system for high-accurate position applications. The random time behaviour with lost packets errors must be considered in the design of localisation algorithms.

\section{Acknowledgement}

The results presented in this paper were developed as part of a study commissioned by Audi AG. The authors would like to thank AUDI AG for supporting their research.

\section{REFERENCES}

[1] J. Huang and H. S. Tan, "Error Analysis and Performance Evaluation of a Future-TrajectoryBased Cooperative Collision Warning System," IEEE Trans. Intell. Transp. Syst., vol. 10, no. 1, pp. 175180, Mar. 2009.

[2] S. Shladover and S.-K. Tan, "Analysis of Vehicle Positioning Accuracy Requirements for Communication-Based Cooperative Collision Warning," J. Intell. Transp. Syst. Technol. Plan. Oper., vol. 10, no. 3, pp. 131-140, Sep. 2006.

[3] J. G. Grimes, "Global Positioning System - Standard Positioning Service - Performance Standard," 2008.

[4] P. Misra and P. Enge, Global Positioning System: Signals, Measurements, and Performance. Lincoln, Mass: Ganga-Jamuna Press, 2010.

[5] K. Ansari, C. Wang, L. Wang, and Y. Feng, "Vehicleto-Vehicle Real-Time Relative Positioning Using 5.9 GHz DSRC Media," in Vehicular Technology Conference (VTC Fall), 2013 IEEE 78th, 2013, pp. $1-7$.

[6] N. Alam, A. Tabatabaei Balaei, and A. G. Dempster, "Relative Positioning Enhancement in VANETs: A Tight Integration Approach," IEEE Trans. Intell. Transp. Syst., vol. 14, no. 1, pp. 47-55, Mar. 2013. 
[7] W. Travis and D. M. Bevly, "Trajectory duplication using relative position information for automated ground vehicle convoys," in Position, Location and Navigation Symposium, 2008 IEEE/ION, 2008, pp. 1022-1032.

[8] VDI/VDE 4000 (1998). General guide to the VDIHandbook reliability engineering. VDI 4001 Blatt 1 , The Association of German Engineers (VDI)

[9] VDI/VDE 2185 (2007). Radio based communication in industrial automation. VDI/VDE 2185, The Association of German Engineers (VDI)

[10] ETSI EN 302571 (V1.1.1): "Intelligent Transport Systems (ITS); Radio communication equipment operating in the $5855 \mathrm{MHz}$ to $5925 \mathrm{MHz}$ frequency band; Harmonized EN covering the essential requirements of article 3.2 of the R\&TTE Directive".

[11] ETSI EN 302663 (V1.2.0): Intelligent Transport Systems (ITS); Access layer specification for Intelligent Transport Systems operating in the $5 \mathrm{GHz}$ frequency band 